\title{
Association Between IL-6 Concentration and Diabetes-Related Variables in DM1 Patients with and without Microvascular Complications
}

\author{
Malgorzata Wegner, ${ }^{1,3}$ Aleksandra Araszkiewicz, ${ }^{2}$ Maria Piorunska-Stolzmann, ${ }^{1}$ \\ Bogna Wierusz-Wysocka, ${ }^{2}$ and Dorota Zozulinska-Ziolkiewicz ${ }^{2}$
}

\begin{abstract}
Interleukin 6 (IL-6) plays an important role in the initiation and acceleration of chronic inflammation and could contribute to development of microvascular complications in patients with type 1 diabetes (DM1). Therefore, this study was aimed to investigate the association between concentration of IL-6 in relation to glucose control, lipid profile, and body mass index (BMI) in 69 DM1 patients subdivided according to the absence or presence of microvascular complications. BMI, level of fasting plasma glucose (FPG), and concentrations of total cholesterol (TCH), LDL cholesterol (LDL-C), and IL-6 were higher in DM1 patients compared to the control group. In DM1 patients, IL-6 concentration was positively correlated with level of FPG, LDL-C, TCH concentrations, and BMI. These correlations were stronger in the subgroup of patients with microvascular complications. In addition, BMI independently influences IL-6 concentration in DM1 patients. In conclusion, elevated IL-6 concentration is associated with diabetes-related variables which could accelerate progression of microvascular complications in DM1 patients.
\end{abstract}

KEY WORDS: BMI; interleukin 6; lipid profile; type 1 diabetes; microvascular complications.

\section{INTRODUCTION}

Chronic vascular complications are the major cause of death in patients with type 1 diabetes (DM1) [1]. However, hyperglycemia seems to be the most critical parameter for development of chronic complications in diabetic patients [2]; the amount of evidences that other factors contribute to this process increase subsequently $[3,4]$.

Recently, it has been shown that patients with DM1 demonstrate higher levels of proinflammatory markers which are associated with atherosclerotic plaque formation and endothelial dysfunction $[5,6]$. These indicate

\footnotetext{
${ }^{1}$ Lipid Metabolism Laboratory, Department of Chemistry and Clinical Biochemistry, Poznan University of Medical Sciences, Grunwaldzka street 6, 60-780 Poznan, Poland

${ }^{2}$ Department of Internal Medicine and Diabetology, Poznan University of Medical Sciences, Mickiewicz street 2, 60-843 Poznan, Poland

${ }^{3}$ To whom correspondence should be addressed at Lipid Metabolism Laboratory, Department of Chemistry and Clinical Biochemistry, Poznan University of Medical Sciences, Grunwaldzka street 6, 60-780 Poznan, Poland. E-mail: malgoweg@ump.edu.pl
}

that an enhanced low-grade inflammatory process may accelerate development of microvascular and macrovascular complications in DM1 patients [7].

One of the markers of inflammation is interleukin 6 (IL-6) [8]. It is a pleiotropic cytokine, produced mostly by adipocytes, fibroblast, endothelial cells, and activated leukocytes and monocytes [9]. IL-6 mainly is known as a chief regulator of acute-phase inflammatory response [10]. However, a critical role of IL-6 in the transformation from acute to chronic inflammation was established as well [11].

In patients with DM1, the concentration of IL-6 has been shown to be normal [12] or higher [13] compared to healthy people. Moreover, some studies suggest that IL-6 participates in the initiation and acceleration of the chronic inflammation process and could contribute to development of micro- and macrovascular complications in diabetic patients $[14,15]$.

Therefore, this study was aimed to investigate the association between concentration of IL-6 in relation to glucose control, lipid profile, and body mass index (BMI) in DM1 patients subdivided according to absence or presence of microvascular complications. 


\section{MATERIALS AND METHODS}

\section{Study Group}

The study group consisted of 69 patients with DM1 recruited by the Department of Internal Medicine and Diabetology at Poznan University of Medical Sciences and participating in the Poznan Prospective Study (NCT01411033) [16]. DM1 was diagnosed according to the criteria from the American Diabetes Association on the basis of classical symptoms, blood glucose concentration higher than $11.1 \mathrm{mmol} / \mathrm{l}$, and C-peptide concentration lower than $0.5 \mu \mathrm{g} / \mathrm{l}$ [17]. All patients were treated with intensive insulin therapy from the onset of the disease. In addition, 13 DM1 patients have been treated with angiotensin-converting enzyme inhibitors (ACE-I) after diagnosis of diabetic kidney disease. The study group was divided according to the absence or presence of chronic microvascular diabetic complications, i.e., retinopathy, diabetic kidney disease, and neuropathy.

Diabetic retinopathy was diagnosed by direct ophthalmoscopy through dilated pupils followed by fundus photography in all patients. Diabetic retinopathy was graded according to the classification of the American Academy of Ophthalmology into no retinopathy; mild, moderate, and severe nonproliferative retinopathy; and proliferative retinopathy [18].

Diabetic kidney disease was detected at the stage of albuminuria (urinary albumin excretion rate between 30 and $300 \mathrm{mg} / 24 \mathrm{~h}$ in two samples collected over a 3month period after exclusion of secondary causes of microproteinuria. Diabetic kidney disease was defined as the presence of albuminuria in connection with diabetes of over 10 years in duration or with diagnosed diabetic retinopathy [19].

Diabetic neuropathy was diagnosed in patients with two or more of the following components: the presence of symptoms of neuropathy, the absence of ankle tendon reflexes, and abnormal scores for pressure and/or vibration perception [17].

The control group consisted of 18 healthy subjects. They were characterized by reference BMI, lipid profile, and glucose concentration. All subjects gave informed consent, and the Ethical Committee of the Poznan University of Medical Sciences approved the study protocol.

\section{Collection of Blood Samples}

Blood samples were collected between 8:00 and 10:00 am after a 12-h fast in tubes without anticoagulant using a standard venipuncture technique. The samples were allowed to clot at room temperature and then centrifuged at 2,000 $\times \mathrm{g}$ for $15 \mathrm{~min}$ to obtain serum. Basic clinical analysis was performed during the same day blood was taken. Subsequently, $1 \mathrm{ml}$ of serum was stored at $-80{ }^{\circ} \mathrm{C}$ until measurement of IL-6 was performed.

\section{Measurements}

The serum concentrations of triglycerides (TG), total cholesterol (TCH), HDL cholesterol (HDL-C), and fasting plasma concentration of glucose (FPG) were determined using the commercially available assay kits (Roche, Switzerland). LDL cholesterol (LDL-C) level was obtained using the following formula: LDL-C=TC-HDL-C $-\mathrm{TG} /$ 5. Postprandial plasma glycemia (PPG) was calculated as the mean of plasma concentration of glucose $2 \mathrm{~h}$ after three main meals in self-control from 3 days before recruitment. The glycated hemoglobin $\mathrm{c}\left(\mathrm{HbA}_{1} \mathrm{c}\right)$ value was measured using high-performance liquid chromatography with the Variant Hemoglobin A1c Program (Bio-Rad Laboratories, Hercules, CA, USA) [20].

The serum IL-6 concentration was measured by enzyme-linked immunosorbent assay (human IL-6 ELISA, Mabtech, Sweden) according to the manufacturer's instruction. The detection limit was $1 \mathrm{pg} / \mathrm{ml}$, intraassay coefficient of variation (CV) was $2.5 \%$, and interassay $\mathrm{CV}$ was $2.8 \%$.

\section{Statistical Analysis}

Data are expressed as mean \pm standard deviation (SD) for normal distribution or median with interquartile range for non-normal distribution. Age, BMI, and systolic and diastolic blood pressure levels were compared between patients with DM1 and the control group by Student's $t$ test. In addition, this test was used to compare diabetes duration between DM1 patients with and without chronic complications. The Mann-Whitney test was performed to detect differences in biochemical parameters and serum IL-6 concentration between patients with DM1 and the control group. Moreover, this test was used to compare levels of PPG and $\mathrm{HbA}_{1} \mathrm{c}$ between DM1 patients without (DM) and with (DMC) chronic microvascular complications. Analysis of variance was used to establish differences of age between patients with and without microvascular complications and the control group. The Kruskal-Wallis test was performed to determine the differences of biochemical parameters and IL-6 concentration between DM1 patients without and with microvascular complications 
and the control group. The Spearman correlation coefficient was used to test the strength of any associations between different variables. In addition, multivariate regression analysis was used to determine which of the selected variables (gender, age, diabetes duration, and BMI level) primarily affect IL-6 concentrations in patients with DM1. A similar analysis was performed to check which parameters of lipid profile independently influence IL-6 concentration in DM1 patients. A $p$ value lower than 0.05 was accepted as statistically significant.

\section{RESULTS}

\section{Clinical Characteristics of DM1 Patients}

There were 24 women and 45 men among Polish patients with DM1. Their mean age was $35.4 \pm 6.3$ years, and the mean diabetes duration was $12.5 \pm 1.5$ years.

In 39 patients (14 women, 25 men), microvascular complications were diagnosed, i.e., retinopathy $(n=15)$, diabetic kidney disease $(n=13)$, and neuropathy $(n=11)$. Therefore, DM1 patients were subdivided into two subgroups according to absence (DM) or presence (DMC) of chronic microvascular complications.

To identify the differences of levels of basic clinical and biochemical variables that could arise from presence of microvascular complications, the two subgroups of DM1 patients were compared with 18 nondiabetic age/gender-matched subjects in the control group (eight women, ten men; $32.1 \pm$ 10.8 years). It was found that DM1 patients without and with microvascular complications did not differ according to TG and HDL concentration and systolic (SBP) and diastolic blood pressure (DBP) compared to healthy subjects. FPG level was much higher in DM1 patients from both subgroups in comparison to healthy people. Glycemic control for most DM1 patients was not satisfactory to maintain good metabolic control. Moreover, DM1 patients from both subgroups had higher BMI than the control group. In addition, DM1 patients with microvascular complications showed higher TCH and LDL-C in comparison to the control group. Significant differences for these parameters were not established between DM1 patients without microvascular complications and the control group. $\mathrm{HbA}_{1} \mathrm{c}$ value was higher in DM1 patients with microvascular complications compared to patients without them (results presented in Table 1).

\section{The Concentration of IL-6 in DM1 Patients}

It was shown that DM1 patients with microvascular complications had higher IL-6 concentration compared to the control group, whereas the concentration of IL-6 did not differ significantly between DM1 patients without microvascular complications and the control group (Fig. 1).

Table 1. Characteristic of DM1 Patients without (DM) and with (DMC) Microvascular Complications and the Control Group

\begin{tabular}{|c|c|c|c|c|}
\hline & $\begin{array}{l}\mathrm{DM} \\
n=30\end{array}$ & $\begin{array}{l}\mathrm{DMC} \\
n=39\end{array}$ & $\begin{array}{l}\text { Healthy group } \\
n=18\end{array}$ & $p$ value \\
\hline $\operatorname{Sex}(\mathrm{F} / \mathrm{M})$ & $10 / 20$ & $14 / 25$ & $8 / 10$ & NS \\
\hline Age (years) & $34.9 \pm 5.4$ & $35.8 \pm 3.4$ & $32.1 \pm 10.8$ & NS \\
\hline BMI $\left(\mathrm{kg} / \mathrm{m}^{2}\right)$ & $24.2 \pm 3.5^{\mathrm{a}}$ & $25.4 \pm 4.1^{\mathrm{a}}$ & $21.1 \pm 1.8$ & 0.0001 \\
\hline FPG (mmol/1) & $8.8(7.3-9.0)^{\mathrm{a}}$ & $9.1(7.6-9.9)^{\mathrm{a}}$ & $4.1(3.2-4.4)$ & 0.000001 \\
\hline PPG (mmol/l) & $8.9(7.2-9.3)$ & $9.2(8.7-9.7)$ & - & NS \\
\hline $\mathrm{HbA}_{1} \mathrm{C}(\%)$ & $7.9(7.1-8.8)$ & $8.6(8.2-9.6)^{b}$ & - & 0.001 \\
\hline $\mathrm{TG}(\mathrm{mmol} / \mathrm{l})$ & $0.9(0.7-1.0)$ & $1.0(0.7-1.5)$ & $1.0(0.7-1.1)$ & NS \\
\hline $\mathrm{TCH}(\mathrm{mmol} / \mathrm{l})$ & $4.4(4.2-4.9)$ & $5.1(4.5-5.6)^{\mathrm{a}}$ & $4.0(3.9-4.5)$ & 0.0004 \\
\hline LDL-C (mmol/l) & $2.6(2.2-3.1)$ & $3.0(2.4-3.4)^{\mathrm{a}}$ & $2.2(1.8-2.3)$ & 0.008 \\
\hline HDL-C (mmol/l) & $1.5(1.5-1.8)$ & $1.6(1.4-1.9)$ & $1.3(0.9-1.4)$ & NS \\
\hline $\mathrm{SBP}(\mathrm{mmHg})$ & $115(110-120)$ & $118(112-130)$ & $105(99-110)$ & NS \\
\hline DBP (mmHg) & $81(79-85)$ & $83(80-85)$ & $75(73-76)$ & NS \\
\hline
\end{tabular}

$B M I$ body mass index, $D B P$ diastolic blood pressure, $F P G$ fasting plasma glucose, $H b A_{1} c$ glycated hemoglobin $\mathrm{A}_{1} \mathrm{c}, H D L-C$ HDL cholesterol, $L D L-C$ LDL cholesterol, $P P G$ postprandial plasma glucose, $S B P$ systolic blood pressure, $T C H$ total cholesterol level, $T G$ triglycerides, $N S$ statistically not significant

${ }^{a}$ Statistical significance versus the control group

${ }^{b}$ Statistical significance versus the group without microvascular complications 


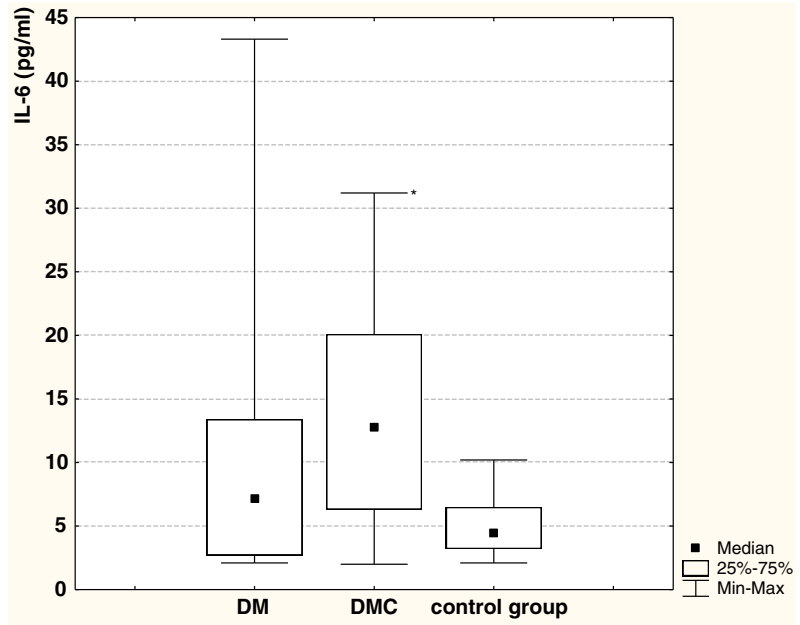

Fig. 1. IL-6 concentration in DM1 patients without (DM) and with (DMC) microvascular complications and the control group; * $p=0.0038$, statistically significant versus the control group.

\section{Correlations of IL-6 Concentration With Diabetes-Related Variables in DM1 Patients}

Another interest of the study was to ascertain the associations between diabetes-related variables and IL-6 concentration in DM1 patients. IL-6 concentration positively correlated with FPG level and LDL-C and TCH concentration ( $r=0.340, r=0.434, r=0.399 ; p<0.05$; $n=69$, respectively). In addition, a strong correlation between IL- 6 concentration and BMI has been found $(r=0.542, p<0.05, n=69)$.

When we looked at these associations separately in DM1 patients without and with microvascular complications, distinct differences were found (Table 2). In addition, in the control group, a positive correlation has been established between level of IL-6 and BMI $(r=0.465, p<0.05, n=18)$. Moreover, the result of multivariate regression analysis showed that BMI inde-

Table 2. The Correlations Between IL-6 Concentration and DiabetesRelated Variables in DM1 Patients Without (DM) and with (DMC) Microvascular Complications

\begin{tabular}{llll}
\hline & IL-6 ratio $(r)$ & & \\
\cline { 2 - 3 } Variable & DM $(n=31)$ & DMC $(n=39)$ & $p$ value \\
\hline FPG $(\mathrm{mmol} / \mathrm{l})$ & $\mathrm{NS}$ & 0.655 & $<0.05$ \\
LDL-C $(\mathrm{mmol} / \mathrm{l})$ & 0.423 & 0.456 & $<0.05$ \\
TCH $(\mathrm{mmol} / \mathrm{l})$ & $\mathrm{NS}$ & 0.402 & $<0.05$ \\
BMI $\left(\mathrm{kg} / \mathrm{m}^{2}\right)$ & 0.427 & 0.738 & $<0.05$ \\
\hline
\end{tabular}

$r$ Pearson's correlation coefficient pendently influences the IL- 6 concentration $(\beta=0.446$, $\left.R^{2}=0.300, p<0.05\right)$ in DM1 patients.

\section{DISCUSSION}

Chronic inflammation could be one of the reasons of endothelial dysfunction and atherosclerotic plaque formation, processes which contribute to the development of micro- and macrovascular complications in patients with diabetes [21]. Elevated IL-6 concentration in the studied group indicates that in DM1 patients, chronic ongoing inflammatory process is present [22]. Moreover, it is shown that not only chronic hyperglycemia but also lipid abnormalities and elevated BMI accelerate development of this process in patients with DM1. It is aligned with other studies, in which similar associations have been found [23, 24].

Observed positive correlation between FPG level and IL-6 serum concentration in DM1 patients is confirmed by the result of an in vitro study, in which has been found that high concentration of glucose induces IL-6 production [4]. This can be explained by the fact, that persistent hyperglycemia contributes to the formation of advanced glycation end products, whose important role in the development of chronic inflammation has already been proved [25].

A strong association between IL-6 concentration and BMI in DM1 patients and in healthy people and the result of multivariable analysis indicate that increased weight strongly influences development of chronic inflammation regardless of any additional clinical or biochemical factors [26]. This finding is coherent with the result of the in vitro study in which enhanced production of IL-6 by human umbilical venous endothelial cells after adding the extract of adipocytes has been shown [27]. Moreover, our results confirm that IL6 is the strong adipocyte-secreted factor [28].

In addition, the positive relation between BMI and IL- 6 concentration and between TCH and IL- 6 serum concentration suggests that IL- 6 can act as a systemic regulator of body mass. It has been shown that IL- 6 can reduce lipoprotein lipase activity [29], which may downregulate adipose tissue triglyceride deposition and promote futile cycling and probably modulates the actions of leptin, a molecule that controls energy balance by causing changes in food intake and physical activity [30]. Therefore, our results show that an elevated level of IL- 6 is associated with diabetes-related variables [31, 32]. 
Moreover, we indicate that not only poor glycemic control [33] but also enhanced production of IL-6 may contribute to the development of microvascular complications in DM1 patients. It could be due to the fact that IL-6 participates in pathogenesis of endothelial dysfunction by stimulation of monocyte chemotactic protein-1 and cell adhesion molecules, such as intercellular adhesion molecule 1 and vascular cell adhesion molecule 1 in endothelial cells [34]. Our proposed explanation is consistent with results of clinical and in vitro studies which found that an enhanced level of IL-6 contributes to the endothelial cell dysfunction [35] and accelerates the development of chronic micro- and macrovascular complications $[13,36]$. Moreover, some clinical results strongly suggest that enhanced IL-6 concentration is most associated with the development of diabetic kidney disease [37]. It seems this could be related to enhanced stimulation of mesangial cell proliferation and interferes with the extracellular dynamics of matrix formation at the podocyte level by IL-6 [38].

However, there is a limitation that needs to be acknowledged and addressed regarding the present study. For assessment of the influence of IL-6 concentration on the acceleration of development of particular chronic complications such as retinopathy, neuropathy, and chronic kidney disease, the size of subgroups of DM1 patients with diagnosed chronic complications should be larger. Moreover, we cannot exclude the potential positive effect of treatment of DM1 patients with diabetic kidney disease with ACE-I on the inhibition of chronic inflammation process, which was already proved in patients with DM2 [39].

In conclusion, our results provide evidence that elevated IL-6 serum concentration is associated with diabetes-related variables, which could accelerate progression of microvascular complications in DM1 patients.

\section{ACKNOWLEDGMENTS}

We wish to thank Dr. Daniel Neddermann for critical revision of the manuscript. We would as well like to kindly thank Mr. Chris Campbell for his help with the English editing of this text.

Conflict of Interest. The authors have no conflicts of interest to declare.
Open Access This article is distributed under the terms of the Creative Commons Attribution License which permits any use, distribution, and reproduction in any medium, provided the original author(s) and the source are credited.

\section{REFERENCES}

1. Abi Khalil, C., R. Roussel, K. Mohammedi, et al. 2012. Causespecific mortality in diabetes: recent changes in trend mortality. Eur J Prev Cardiol 19: 374-381.

2. Brownlee, M. 2001. Biochemistry and molecular cell biology of diabetic complications. Nature 414: 813-820.

3. Wegner, M., A. Araszkiewicz, A. Pioruńska-Mikołajczak, et al. 2009. The evaluation of IL-12 concentration, PAF-AH, and PLA (2) activity in patients with type 1 diabetes treated with intensive insulin therapy. Clinical Biochemistry 42: 1621-1627.

4. Marcovecchio, M.L., R.N. Dalton, A.T. Prevost, et al. 2009. Prevalence of abnormal lipid profiles and the relationship with the development of microalbuminuria in adolescents with type 1 diabetes. Diabetes Care 32: 658-663.

5. Aguiar, L.G., N.R. Villela, and E. Bonskela. 2007. Microcirculation in diabetes implications for chronic complications and treatment of the disease. Arg Bras Endorcrinol Metabol 51: 204-211.

6. Devaraj, S., A.T. Cheung, I. Jialal, et al. 2007. Evidence of increased inflammation and microcirculatory abnormalities in patients with type 1 diabetes and their role in microvascular complications. Diabetes 56: 2790-2796.

7. Astrup, A.S., L. Tarnow, L. Pietraszek, et al. 2008. Markers of endothelial dysfunction and inflammation in type 1 diabetic patients with or without diabetic nephropathy followed for 10 years. Diabetes Care 31: 1170-1176.

8. Croker, B.A., H. Kiu, M. Pellegrini, et al. 2012. IL-6 promotes acute and chronic inflammatory disease in the absence of SOCS3. Immunology and Cell Biology 90: 124-129.

9. Waetzig, G.H., and S. Rose-John. 2012. Hitting a complex target: an update on interleukin-6trans-signalling. Expert Opinion on Therapeutic Targets 16: 225-236.

10. Mihara, M., M. Hashizume, H. Yoshida, et al. 2012. IL-6/IL-6 receptor system and its role in physiological and pathological conditions. Clinical Science 122: 143-159.

11. Gabay, C. 2006. Interleukin-6 and chronic inflammation. Arthritis Research \& Therapy 8(Suppl 2): 3.

12. Kulseng, B., L. Vatten, and T. Espevik. 1999. Soluble tumor necrosis factor receptors in sera from patients with insulindependent diabetes mellitus: relations to duration and complications of disease. Acta Diabetologica 36: 99-105.

13. Targher, G., L. Zenari, L. Bertolini, et al. 2001. Elevated levels of interleukin-6 in young adults with type 1 diabetes without clinical evidence of microvascular and macrovascular complications. Diabetes Care 24: 956-957. Letter.

14. Saraheimo, M., A.M. Teppo, C. Forsblom, et al. 2003. Diabetic nephropathy is associated with low-grade inflammation in type 1 diabetic patients. Diabetologia 46: 1402-1407.

15. Jialal I, Kaur H. 2013. The role of toll-like receptors in diabetesinduced inflammation: implications for vascular complications. Current Diabetes Reports (In press)

16. Araszkiewicz, A., D. Zozulinska-Ziolkiewicz, M. Trepinska, et al. 2008. Knowledge after five-day teaching program in intensive insulin therapy performed at the onset of type 1 diabetes influence the development of late diabetic complications. Diabetes Research and Clinical Practice 81: 61-67. 
17. American Diabetes Association. 2007. Standards of medical care in diabetes-2007. Diabetes Care 30: 4-41.

18. Wilkinson, C.P., F.L. Ferris, R.E. Klein, et al. 2003. Global Diabetic Retinopathy Project Group. Proposed international clinical diabetic retinopathy and diabetic macular edema disease severity scales. Ophthalmology 110: 1677-1682.

19. KDOQI. 2007. KDOQI clinical practice guidelines and clinical practice recommendations for diabetes and chronic kidney disease. American Journal of Kidney Diseases 49: 12-154.

20. Jeppsson, J.O., U. Kobold, J. Barr, et al. 2002. International Federation of Clinical Chemistry and Laboratory Medicine (IFCC). Approved IFCC reference method for the measurement of $\mathrm{HbA} 1 \mathrm{c}$ in human blood. Clinical Chemistry and Laboratory Medicine 40: 78-79.

21. Hartge, M.M., T. Unger, and U. Kintscher. 2007. The endothelium and vascular inflammation in diabetes. Diabetes \& Vascular Disease Research 4: 84-88.

22. Fisman, E.Z., and A. Tenenbaum. 2010. The ubiquitous interleukin-6: a time for reappraisal. Cardiovascular Diabetology 11: 9-62.

23. Navarro, J.F., and M. Carmen. 2005. Role of inflammation in diabetic complications. Nephrology, Dialysis, Transplantation 20: 2601-2604.

24. Munch, I.C., M. Larsen, L. Kessel, et al. 2011. Cumulative glycemia and microangiopathy in subjects with impaired glucose regulation in the Inter 99 study. Diabetes Research and Clinical Practice 91: 226-232.

25. Nevado, J., C. Peiró, S. Vallejo, et al. 2005. Amadori adducts activate nuclear factor-kappaB-related proinflammatory genes in cultured human peritoneal mesothelial cells. British Journal of Pharmacology 146: 268-279.

26. Illán-Gómez, F., M. Gonzálvez-Ortega, I. Orea-Soler, et al. 2012. Obesity and inflammation: change in adiponectin, C-reactive protein, tumour necrosis factor-alpha and interleukin-6 after bariatric surgery. Obesity Surgery 22: 950-955.

27. Sommer, G., S. Kralisch, V. Stangl, et al. 2009. Secretory products from human adipocytes stimulate proinflammatory cytokine secretion from human endothelial cells. Journal of Cellular Biochemistry 106: 729-737.

28. Mohamed-Ali, V., S. Goodrick, and A. Rawesh. 1997. Subcutaneous adipose tissue releases interleukin-6, but not tumor necrosis factor-alpha, in vivo. Journal of Clinical Endocrinology and Metabolism 82: 4196-4200.
29. Greenberg, A.S., R.P. Nordan, J. McIntosh, et al. 1992. Interleukin 6 reduces lipoprotein lipase activity in adipose tissue of mice in vivo and in 3T3-L1 adipocytes: a possible role for interleukin 6 in cancer cachexia. Cancer Research 52: 4113-4116.

30. Campfield, L.A., F.J. Smith, Y. Guisez, et al. 1995. Recombinant mouse OB protein: evidence for a peripheral signal linking adiposity and central neural networks. Science 269: 546-549.

31. Fawaz, L., A.E. Elwan, Y.H. Kamel, et al. 2009. Value of Creactive protein and IL-6 measurements in type 1 diabetes mellitus. Arch Med Sci 3: 383-390.

32. Nakamura, N., G. Hasegawa, H. Obayashi, et al. 2003. Increased concentration of pentosidine, an advanced glycation end product, and interleukin-6 in the vitreous of patients with proliferative diabetic retinopathy. Diabetes Research and Clinical Practice 61: 93-101.

33. Nalysnyk, L., M. Hernandez-Medina, and G. Krishnarajah. 2010. Glycaemic variability and complications in patients with diabetes mellitus: evidence from a systematic review of the literature. Diabetes, Obesity \& Metabolism 12: 288-298.

34. Lau, D.C., B. Dhillon, H. Yan, et al. 2005. Adipokines: molecular links between obesity and atherosclerosis. American Journal of Physiology-Heart and Circulatory Physiology 288: 2031-2041.

35. Georgescu, A., N. Alexandru, E. Andrei, et al. 2012. Circulating microparticles and endothelial progenitor cells in atherosclerosis: pharmacological effects of irbesartan. Journal of Thrombosis and Haemostasis 10: 680-691.

36. Kolluru, G.K., S.C. Bir, and C.G. Kevil. 2012. Endothelial dysfunction and diabetes: effects on angiogenesis, vascular remodeling, and wound healing. Int J Vasc Med 2012: 918267.

37. Jisieike-Onuigbo, N.N., E.I. Unuigbe, O.A. Kalu, et al. 2011 Prevalence of dyslipidemia among adult diabetic patients with overt diabetic nephropathy in Anambra state south-east Nigeria. Nigerian Journal of Clinical Practice 14: 171-175.

38. Dalla Vestra, M., M. Mussap, P. Gallina, et al. 2005. Acute-phase markers of inflammation and glomerular structure in patients with type 2 diabetes. Journal of the American Society of Nephrology 16: $78-82$.

39. Marketou, M.E., E.A. Zacharis, S. Koukouraki, et al. 2008. Effect of angiotensin-converting enzyme inhibitors on systemic inflammation and myocardial sympathetic innervation in normotensive patients with type 2 diabetes mellitus. Journal of Human Hypertension 22: 191-196. 